\title{
Picture of glioma stem cells has become a Notch brighter
}

\author{
Prahlad Parajuli, Sandeep Mittal \\ Department of Neurosurgery, Wayne State University School of Medicine \& Karmanos Cancer Institute, Detroit, MI 48201, USA \\ Correspondence to: Prahlad Parajuli. Department of Neurosurgery, Wayne State University School of Medicine \& Karmanos Cancer Institute, Detroit, \\ MI 48201, USA. Email: pparajuli@med.wayne.edu. \\ Comment on: Man J, Yu X, Huang H, et al. Hypoxic Induction of Vasorin Regulates Notch1 Turnover to Maintain Glioma Stem-like Cells. Cell Stem \\ Cell 2018;22:104-118.e6.
}

Received: 22 October 2018; Accepted: 09 November 2018; Published: 21 November 2018.

doi: $10.21037 /$ sci.2018.11.02

View this article at: http://dx.doi.org/10.21037/sci.2018.11.02

Solid tumors often behave like an organ system, comprised of rapidly proliferating cancer cells and a variety of stromal components, including endothelial cells (ECs), fibroblasts, and immune cells. Similar to an organ system, all tumors, including malignant gliomas, also contain a small population of cancer stem cells (CSCs) with adult stem cell-like characteristics, namely, pluripotency and capacity for self-renewal. CSCs are quiescent and have low immunogenicity - features that help them evade destruction by chemotherapy and immunotherapy, respectively. Identification of novel molecular cues that regulate maintenance (self-renewal) of CSCs could help develop new therapeutic interventions for complete eradication of cancer and avoid recurrence.

In a tumor, the various cellular components provide physical and chemical cues which help regulate maintenance of CSC population, induce proliferation of cancer cells, and promote heterogeneity among cancer cells (1) (Figure 1). It has been established that the fate of CSCs are determined by paracrine or juxtacrine signalling from neighboring cells (2). In malignant gliomas, the glioma stem cells (GSCs) are often reported to be located in hypoxic and perivascular areas. Zhu et al. have shown that, in perivascular areas, Notch ligands in ECs could increase GSC phenotype in neighboring cancer cells while also enhancing their capacity for self-renewal (3).

The Notch signalling pathway is evolutionarily conserved in all metazoans. It comprises of one receptor protein in Drosophila, while humans have four receptor proteins (Notch 1-4) and two classes of ligand molecules (Delta-like and Serrate/Jagged) (4). Canonical Notch signalling plays a critical role in embryonic development and organogenesis. On the other hand, both canonical and non-canonical
Notch signalling have been implicated in shaping tumorstromal interaction and regulating CSC maintenance $(4,5)$. Notch ligands expressed by ECs have been known to influence Notch signalling and cell fate in neighboring cancer cells $(6,7)$. However, the exact molecular mechanism of GSC-intrinsic Notch activity, especially in hypoxic areas, remains unknown. A recent publication by Man et al. has provided a breakthrough in this direction by identifying a novel molecular mechanism by which Notch signalling regulates the fate of GSCs (8). The report very elegantly and convincingly demonstrates how hypoxic condition induces expression of Vasorin in GSCs via activation of HIF-1 $\alpha /$ STAT 3 coactivator complex and Vasorin, in turn, stabilizes Notch in the membrane and prevents it from Numb-mediated ubiquitylation and lysosomal degradation (8).

Immune cells comprise an integral component of the tumor 'organ'. Recent successful outcomes of immunotherapy in various cancers, especially with checkpoint inhibitors, have brought immunotherapy to the forefront of cancer therapeutics. Moreover, awarding the 2018 Nobel Prize in Medicine to Drs. James Alison and Tasuku Honjo for their discovery of immune checkpoint molecules have brought further attention to the field. However, in majority of cases, the remission following immunotherapy is temporary and CSCs have been widely implicated in tumor recurrence. There is limited knowledge about interplay between CSCs and stromal immune cells; this is especially relevant in malignant gliomas. Studies from our group and others have implicated cytokinemediated activation of STAT- 3 and NF- $\kappa \mathrm{B}$ transcription factors in the maintenance and self-renewal of GSCs $(9,10)$. In this context, activation of Notch signalling or 


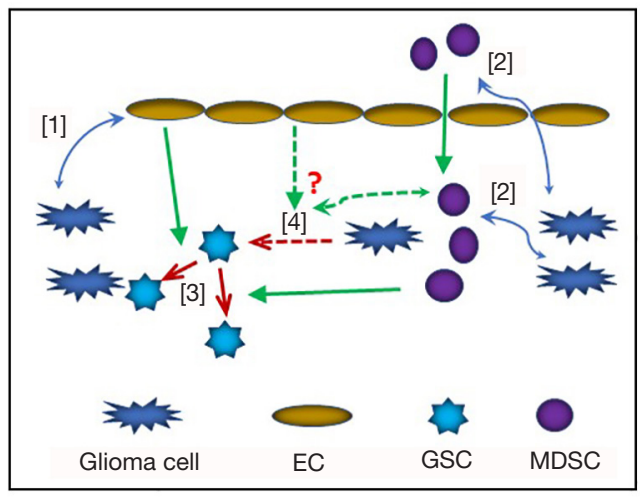

Figure 1 Interplay among glioma cells, GSCs, ECs and MDSCs involving Notch-Notch ligand interaction. Glioma cells induce angiogenesis and EC proliferation [1]. Glioma cells also recruit and stimulate MDSCs in the tumor microenvironment [2]. The ECs and MDSCs in turn, induce GSC self-renewal and maintenance [3]. Furthermore, ECs and MDSCs could potentially induce EMT and stemness in neighboring tumor cells [4]. All these events involve either direct (canonical) or indirect (non-canonical) activation of Notch signalling molecules. GSCs, glioma stem cells; ECs, endothelial cells; MDSCs, myeloid derived suppressor cells.

dysregulation thereof in the stromal immune cells have also been implicated in shaping anti-tumor or pro-tumor microenvironment via different intracellular pathways, which often tend to be context-dependent (7). On the other hand, Notch ligand Jag2 expressed on myeloid derived suppressor cells (MDSCs) has been shown to induce epidermal-mesenchymal transition in colorectal cancer (11). Moreover, STAT-3 and NF-кB signalling pathways have been shown to cross talk with and regulate Notch signalling in GSCs $(12,13)$. In hypoxic conditions, HIF- $1 \alpha$ have been shown to significantly enhance the expression of checkpoint molecule PDL1 in tumor cells as well as in tumor associated macrophages (TAM) and MDSCs (14). Therefore, immunotherapy, combined with strategies targeting GSC-immune interaction involving HIF-1/Notch signalling could lead to better clinical outcomes in solid tumors, including high-grade gliomas.

A few fundamental questions about CSCs continue to trouble cancer researchers. Examples include, whether there is a pre-existing, finite population of CSCs in tumors and whether, under certain conditions, 'differentiated' tumor cells can be induced to acquire CSC phenotype. A study by Suva et al. identified four transcription factors (namely, POU3F2, SOX2, SALL2, and OLIG2), simultaneous activation of which could induce 'stemness' in gliomas (15). Since GSCs are usually found in hypoxic areas, it is not unreasonable to assume that hypoxia could induce GSC phenotype in glioma cells.

Pertinent to this topic, Singh et al. first described GSCs as a population of $\mathrm{CD} 133^{+}$cells (16) and hypoxic condition has been demonstrated to induce expansion of CD133+ glioma stem-like cells (17). Moreover, CD133 is a hypoxiainducible protein and recent studies have illustrated the possibility of inducing stem cell-like (CD133+) phenotype and self-renewing capacity in differentiated (CD133-) glioma cells under hypoxic conditions $(18,19)$. The study by Man et al. provides detailed molecular mechanism regarding how hypoxic condition induces GSC self-renewal and maintenance via Notch activation, but it does not confirm that hypoxia could actually induce 'stemness' in gliomas. More advances must be made in this direction before we find an answer to this conundrum.

\section{Acknowledgements}

None.

\section{Footnote}

Conflicts of Interest: The authors have no conflicts of interest to declare.

\section{References}

1. Lytle NK, Barber AG, Reya T. Stem cell fate in cancer growth, progression and therapy resistance. Nat Rev Cancer 2018;18:669-80.

2. Xie Q, Mittal S, Berens ME. Targeting adaptive glioblastoma: an overview of proliferation and invasion. Neuro Oncol 2014;16:1575-84.

3. Zhu TS, Costello MA, Talsma CE, et al. Endothelial cells create a stem cell niche in glioblastoma by providing NOTCH ligands that nurture self-renewal of cancer stemlike cells. Cancer Res 2011;71:6061-72.

4. Kopan R, Ilagan MX. The canonical Notch signalling pathway: unfolding the activation mechanism. Cell 2009;137:216-33.

5. Ayaz F, Osborne BA. Non-canonical notch signalling in cancer and immunity. Front Oncol 2014;4:345.

6. Takebe N, Miele L, Harris PJ, et al. Targeting Notch, Hedgehog, and Wnt pathways in cancer stem cells: clinical update. Nat Rev Clin Oncol 2015;12:445-64. 
7. Meurette O, Mehlen P. Notch Signaling in the Tumor Microenvironment. Cancer Cell 2018;34:536-48.

8. Man J, Yu X, Huang H, et al. Hypoxic Induction of Vasorin Regulates Notch1 Turnover to Maintain Glioma Stem-like Cells. Cell Stem Cell 2018;22:104-18.e6.

9. Parajuli P, Anand R, Mandalaparty C, et al. Preferential expression of functional IL-17R in glioma stem cells: potential role in self-renewal. Oncotarget 2016;7:6121-35.

10. Haftchenary S, Luchman HA, Jouk AO, et al. Potent Targeting of the STAT3 Protein in Brain Cancer Stem Cells: A Promising Route for Treating Glioblastoma. ACS Med Chem Lett 2013;4:1102-7.

11. Caiado F, Carvalho T, Rosa I, et al. Bone marrowderived CD11b+Jagged2 + cells promote epithelial-tomesenchymal transition and metastasization in colorectal cancer. Cancer Res 2013;73:4233-46.

12. Garner JM, Fan M, Yang CH, et al. Constitutive activation of signal transducer and activator of transcription 3 (STAT3) and nuclear factor kappaB signalling in glioblastoma cancer stem cells regulates the Notch pathway. J Biol Chem 2013;288:26167-76.

13. Yan D, Hao C, Xiao-Feng L, et al. Molecular mechanism of Notch signalling with special emphasis on microRNAs:
Implications for glioma. J Cell Physiol 2018;234:158-70.

14. Noman MZ, Desantis G, Janji B, et al. PD-L1 is a novel direct target of HIF-1alpha, and its blockade under hypoxia enhanced MDSC-mediated T cell activation. J Exp Med 2014;211:781-90.

15. Suva ML, Rheinbay E, Gillespie SM, et al. Reconstructing and reprogramming the tumor-propagating potential of glioblastoma stem-like cells. Cell 2014;157:580-94.

16. Singh SK, Hawkins C, Clarke ID, et al. Identification of human brain tumour initiating cells. Nature 2004;432:396-401.

17. Soeda A, Park M, Lee D, et al. Hypoxia promotes expansion of the CD133-positive glioma stem cells through activation of HIF-1alpha. Oncogene 2009;28:3949-59.

18. Wang P, Lan C, Xiong S, et al. HIF1alpha regulates single differentiated glioma cell dedifferentiation to stem-like cell phenotypes with high tumorigenic potential under hypoxia. Oncotarget 2017;8:28074-92.

19. Lee G, Auffinger B, Guo D, et al. Dedifferentiation of Glioma Cells to Glioma Stem-like Cells By Therapeutic Stress-induced HIF Signalling in the Recurrent GBM Model. Mol Cancer Ther 2016;15:3064-76. doi: $10.21037 /$ sci.2018.11.02

Cite this article as: Parajuli P, Mittal S. Picture of glioma stem cells has become a Notch brighter. Stem Cell Investig 2018;5:42. 